\title{
Needs, Demands and Reality of People with Neuromuscular Disorders Users of Wheelchair
}

\author{
Thais Pousada $^{1 *}$, Javier Pereira ${ }^{1}$, Emiliano Díez ${ }^{2}$, Betania Groba ${ }^{1}$, Laura Nieto ${ }^{1}$ and Alejandro Pazos ${ }^{1}$ \\ ${ }^{1}$ University of a Coruña, Research Group RNASA-IMEDIR, Spain \\ ${ }^{2}$ University of Salamanca, Research Group INICO, Spain
}

*Corresponding author: Thais Pousada, University of a Coruña, Research Group RNASA-IMEDIR, Spain

Submission: 眥 July 28, 2017; Published: 眥 November 13, 2017

\begin{abstract}
Background: The progressive nature of neuromuscular disease (NMD) results in a reduction in mobility: a person's ability to move about, or locomotion. Wheelchair is an assistive technology device (AT) that is fundamental to provide a greater degree of independence in mobility to those affected by an NMD, and its use is mediated by factors related to context, activity, and degree of participation.
\end{abstract}

Purpose: To provide an overview of the profile of participants (persons with neuromuscular disorder user of wheelchair)

i. To determine the characteristics of wheelchair used by participants

ii. To determine the impact of wheelchair in life of people with NMD.

Methodology: Cross-sectional study, with a transversal design was employed. Sample was formed by 36 men and 24 women with Neuromuscular Disease (NMD), user of wheelchair, and their caregiver. To obtain information about activities, participation and contextual factors, an original questionnaire was employed, and to evaluate the impact of wheelchair the Psychosocial Impact of Assistive Devices Scale (PIADS) was applied.

Results: The results show that, in general, psychosocial impact associated to wheelchair use is positive, with the type of wheelchair, correct matching between AT-person and mobility independence as the main determinants.

Conclusion: Knowledge of how the wheelchair is incorporated into a user's daily life will help him/her to reach his/her objectives and expectations, and to establish the social and personal benefits derived from its use. Independence in personal mobility is the main factor determining a positive impact on the quality of life associated with wheelchair use.

Keywords: Neuromuscular disorders; Occupational therapy; Wheelchair; Independencey

\section{Introduction}

Neuromuscular Disorders are a group of neurological pathologies, with more than 150 different diagnoses. The majority is genetics, but some of them can be acquired. They affect to the muscle, the neuromuscular union, the peripheral nerve or the spinal motoneuron [1]. In general, they are chronicle, progressive and neurodegenerative pathologies. They can be diagnosed at different range of age, affecting people in various stages of their vital cycles. Their main clinical characteristic is the loss of muscle strength, that can be accompanied of muscular atrophy, myotonia, cramps, muscle contractures, pain and, sometimes, sensitive problems [2,3].

Prevalence and incidence of these diseases depends on group of diagnosis and available registers in each country. To make an idea about data, for example, it estimated that Duchenne Muscular Dystrophy (DMD), affects mainly to men, and it has an incidence of 1 of 3500 newborns [4]. Symptoms affect the skills of person in different ways, and it conditions her/his independence to performance activities of daily life (AVD). Once the diagnosis is established, patients with limitations in activities and participation problems and a need for help rehabilitation's professionals are increased [5].

Also the need of resource supports, like assistive technology, home adjustments and/or support from caregiver is evident Pousada et al. [6]. There is not curative treatment for NMD, but that doesn't mean that they don't have treatment. Rehabilitation constitutes the best approach to face and to diminish the progression of the disease. It has to include recurrent assessments regarding their ability to ambulate, to manage their work and hometask, and contemplates the need of use any assistive technology [7]. One of the most used assistive technologies by this population is wheelchair. Its use is conditioned by lower limb's strength and it can be sporadic in the initial stages. The increase of frequency is derived from disease's progression. Although certain benefits of wheelchair use (facilitating independence in locomotion, promotion of personal autonomy or maintenance of social participation), 
person could find several negative points, as the acceptance of the wheelchair implies to assume that disease is progressing, the social stigma of disability of the failure to meet person's needs, due to bad prescription [8-10].

Although it is clear that people with NMD need a complete and multidisciplinary rehabilitation intervention, no guidelines or criteria exist for referral patients to physical therapy, occupational therapy or speech therapy. Studies have not enough evidence to assume the correct management of these cases [1]. So, in order to identify the main problems in functional status or quality of life of people with NMD, and to improve rehabilitation strategies to this population, it is necessary to carry out descriptive studies. That will allow identifying variables of interest and influential factors that could be oriented the intervention's approach. Moreover, the application of outcome measures is a strategy that helps to highlight the real efficacy and efficiency derived from different resources for intervention, among that, the use of assistive technology to maintain independence.

This article offers the results from a descriptive study carried out with a sample of persons with NMD, in a region of Spain. Proposed goals were:

i. To provide an overview of the profile of participants (persons with neuromuscular disorder user of wheelchair)

ii. To determine the characteristics of wheelchair used by participants

iii. To determine the impact of wheelchair in life of people with NMD.

\section{Methodology \\ Design}

Cross-sectional study design was applied. Research has done in Galicia, region of Spain, during a period of 16 months.

\section{Sample and selection of participants}

Participants are collected from a non-profit organization of that region of Spain. That was conditioned by the fact that there is not official register of people affected by NMD in neither Department of Health nor official consensus data. Sample was formed by 60 persons with NMD (men=36; women=24), that met with the follow inclusion criteria:

a. Having a known or probable diagnosis of a neuromuscular disease

\section{b. Residing in Galicia}

c. Age greater than or equal to 7 years old (range of age was limit in 7 years because it is determine by one of assessment tools applied)

d. Using a wheelchair on a regular basis.

All participants given their consent to get involved in the study, and all ethical criteria established by the regional research committee were respected.

\section{Variables and measures}

The characteristics studied in present research can be divided into three groups:

a. Personal factors: Socio demographical and clinical data, and information about his/her activity and participation.

b. Features of wheelchair: physical characteristics, accessories and type of use, as well as, the influence of his/her wheelchair in the life of person.

c. Environmental Factors: Here support resources used by person with NMD were included. Among that, it highlights the need to use of assistive technology, adjustments at home and help from caregiver.

To collect that data, researchers have used few instruments:

General questionnaire: This tool was elaborated by research group in order to collect the data about demographics, activity and participation in occupational areas (instrumental activities of daily life, work, education, leisure, and community participation), a questionnaire was developed by our research and information about environmental factors of person, specially, social and physical contexts. Moreover, it incorporated a section to gather the features of wheelchair used by person to complete this one, interviews with both the patient and the caregiver were done. As this specific questionnaire has specifically been made for the present study, its psychometric properties are not available. Nevertheless, before applying this tool, a previous testing was performed with three users.

Functional Independence Measure (FIM): This tool was used to determine the level of personal autonomy in performing activities of daily living [11]. The questionnaire assesses six areas of functioning across 18 items: self-care, sphincter control, mobility, locomotion, communication and social cognition. These areas are grouped into two domains: Motor (13 items) and Cognitive (5 items). Each item is scored using the seven-point Likert scale, ranging from 1 (total assistance is needed) to 7 (performance without any support) [12]. The maximum score is 126 , indicating functional independence, and the lowest score is 18 , corresponding to maximum dependence. Validity, reliability and sensitivity to change have been well established [13-15]. FIM has been validated in Spanish and this version is that has been applied in present study [16].

Psychosocial Impact of Assistive Technology Scale (PIADS): This is a 26 item self-report questionnaire designed to assess the effects of an assistive device on functional independence, wellbeing and quality of life. To assess that dimension, the questionnaire includes three subscales: competence (12 items), adaptability (6 items) and self-esteem (8 items) [17].

Each item is scored separately on a 7 point Likert Scale (from -3 (very negative) to +3 -very positive). Psychometric properties of PIADS have been demonstrated Day et al. [18], Jutai et al. [19], Jutai et al. [20] and it has validated into Spanish context. 


\section{Procedure}

\section{Study integrated by three consecutive steps}

i. Contact with participants: First, and to check that possible participants met with inclusion criteria, researcher contacted with all persons included in NGO's database (249 records). If individuals had the minimal characteristics to be involved into research, they obtained the complete information and their informed consent was solicited. At this moment, researchers fixed, according to the person and his/her family, the date to have an appointment with them for applying assessment tools.

ii. Assessment process: This step contemplated the performance of interview with person with neuromuscular disorder and his/her relative/caregiver. This meeting was done at participant's home, preferably, but if it was not possible, the interview was taken place at the center of NGO. Personal meetings were performance with 59 participants and 38 caregivers. The rest of participants that complete the sample (1 person with NMD and 2 caregivers), were interviewed by phone, due to the impossibility to arrange face-to-face meeting. During interview, participants could clarify any doubt about study and research gives them all information. Assessment tools (general questionnaire, FIM and PIADS) were applied in the context of a semi-structured interview.

iii. Feedback and data recording: Once all meetings with participants finished, researchers were recording data into statistical software, digitalizing the questionnaires. At the same time, descriptive and bivariate analysis was applied. Then, participants received feedback from researchers in order to give them the information about their specific results and a brief report about study's findings.

\section{Data Analysis}

All questionnaires were digitalized and codified to maintain the confidentiality of personal data. Statistics descriptive analysis was applied through SPSS v.20 for Windows®: Quantitative variables are expressed as mean (SD), median, and range; qualitative variables are showed with their frequency and percentage. Bavarian analysis was done to determine the presence of relationships with highlighted significance between variables. The non-parametric tests were used because we could not assume that the data fit a normal distribution. So, Spearman's correlation coefficient for ranked data (rho) was used to examine the relationship between quantitative variables. To make the association between qualitative variables, a $\chi 2$ measure was used. The combination of qualitative and quantitative variables was studied with U Mann Whitney Test or Kruskal-Wallis test, according type of variable. The level of significance was set to $\mathrm{p} \leq 0.05$.

\section{Results}

Sample included 60 persons with NMD, 60\% (N=36) were men and the $40 \%$, women. The mean age of the sample was 35.15 years (range 7-71, SD 17.1). The 20\% were married at the moment of research, and only five persons were working (10.8\%). Thirty-one persons from sample are used a powered wheelchair, while the 48.3\% used any manual wheelchair: six persons had non-propelled wheel chair and 23 was able to drive a manual self-propelled wheelchair. From sample, 43 persons with NMD (76.7\%) needed the support from family member as caregiver, and other 14 persons had a personal assistant (not relative). For two of these people, the support from caregiver was less than two hours per day. Then, the results are showed divided according the three groups of variables previously commented.

\section{Personal factors, activity \& participation}

Demographical and clinical data are showed in Table 1 . In general, sample has a heterogeneous profile, highlighting the high level of disability $79 \%$ over 100 . The most numerous group of diagnosis was Muscular dystrophy (58\%), followed by group of Spinal Muscular Atrophy (12\%). The $47 \%$ of sample were not receiving any treatment of rehabilitation, while the $44 \%$ received session of physical therapy. With respect to independence in ADL, assessed with FIM, is obtained that there is a heterogenic profile of independence. In general, participants have a good cognitive level, and the activities with less level of independence are: bath/shower, dressing, and transfers.

Table 1: Demographical and clinical characteristics of participants.

\begin{tabular}{|c|c|c|c|}
\hline \multicolumn{2}{|c|}{ Qualitative Variables } & $\mathbf{N}$ & \multirow{2}{*}{$\begin{array}{c}\text { \% } \\
60 \%\end{array}$} \\
\hline Cond & Men & 36 & \\
\hline Gender & Women & 24 & $40 \%$ \\
\hline \multirow{4}{*}{ Marital Status } & Single & 48 & $80 \%$ \\
\hline & Married & 12 & $20 \%$ \\
\hline & Divorced & 0 & 0 \\
\hline & Widower & 0 & 0 \\
\hline \multirow{4}{*}{ City } & $\begin{array}{l}\text { City with more than } 200.000 \text { inhabitant } \\
\text { (inhab) }\end{array}$ & 18 & $30 \%$ \\
\hline & City between 85.000 and 199.999 inhab. & 10 & $16.70 \%$ \\
\hline & City between 40.000 and 84.999 inhab. & 6 & $10 \%$ \\
\hline & Town with less than 39.999 inhab. & 26 & 43.30 \\
\hline
\end{tabular}




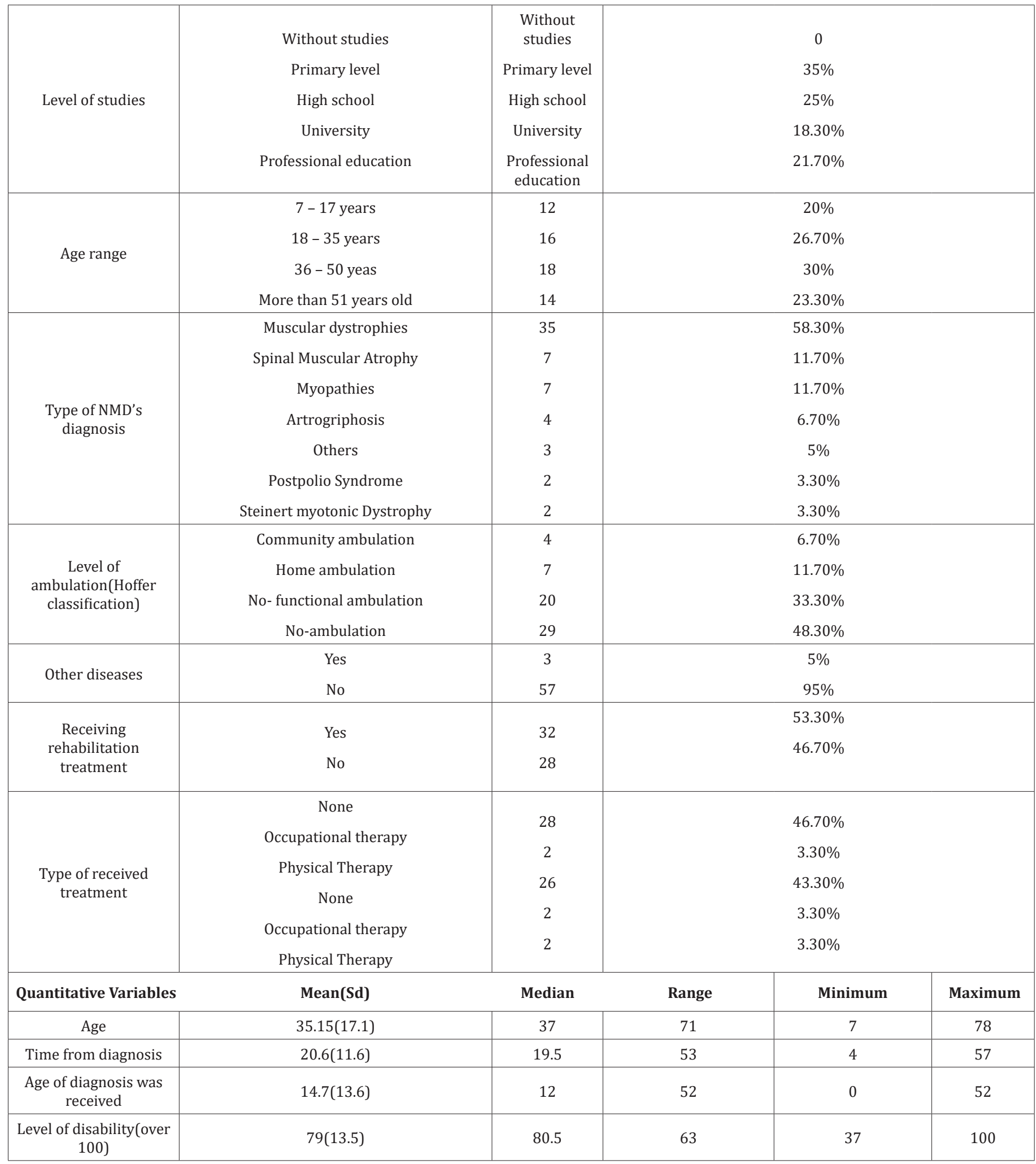

In the Figure $1 \& 2$, the level of independence in locomotion is showed to type of wheelchair and to level of ambulation. Eighteen participants were studying during research, and 16 of them used the wheelchair at education's center. Only one user was in special education mode. The persons of sample who had a minimal age to work (older than 16 and younger than 65 ) were 46 , but only 5 of them were working at this moment. The main cause of don't have a job was the legal recognition for incapacity to work, according Spanish laws. With respect to leisure activities, the $81 \%$ used the wheelchair during performance of those activities, and so, the $91.7 \%$ had to change their habits of leisure due to the progression of disease, and the presence of architectonical barriers. 


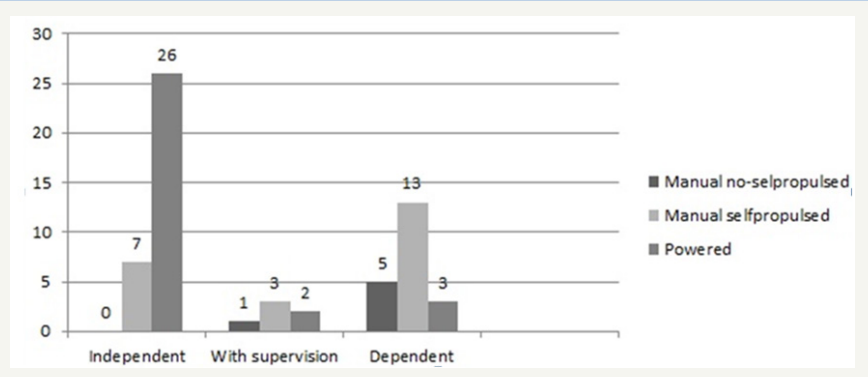

Figure 1: Level of Independence in locomotion.

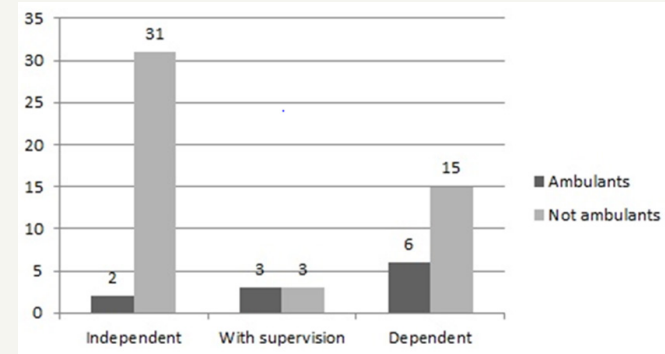

Figure 2: Level of independence in locomotion.

\section{Features of wheelchair}

Almost the half of sample was used a powered wheelchair (31 persons), and the others had a manual wheelchair $(\mathrm{N}=29)$. The main characteristics related to profile of use and the provision systems are showed in Table 2 . It highlight the wide range for time elapsed since the first wheelchair (range=36 years) and the age to start to use a wheelchair (range $=71$ years) between participants.
Only three participants were using the wheelchair exclusively at home (a manual wheelchair). Twenty-one persons used it outdoors (Powered=8; manual=13), and the rest, were using their device in both spaces (outdoors and indoors). With respect to the main problems derived from the use of wheelchair, (Figure $3 \& 4$ ), showed that information to regarding the two types of wheelchair. In both cases, the weight gain was the main negative factor.

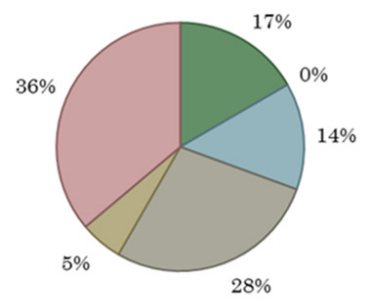

$\square$ Debilidad
$\square$ UPP
$\square$ Dolor
$\square$ Escoliosis / Contracturas
$\square$ Problemas Respiratorios
$\square$ Aumento de peso

Figure 3: Associated problems related to use of manual wheelchair.

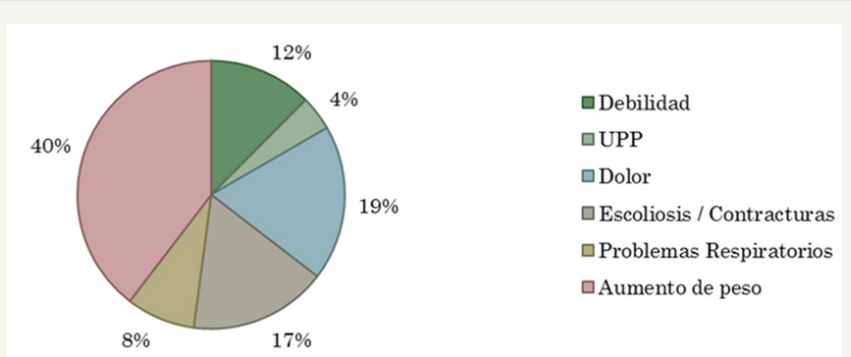

Figure 4: Associated problems related to use of electric wheelchair.

Table 2: Characteristics of wheelchair.

\begin{tabular}{|c|c|c|}
\hline Quantitative Variables & Median(SD) & Range \\
\hline Number of used wheelchair & $2.85(1.52)$ & 7 \\
\hline number wheelchair using right now & $1.35(0.48)$ & 1 \\
\hline Age of starting to use the first wheelchair(years) & $22.8(15.83)$ & 71 \\
\hline Time from the first wheelchair(years) & $12.35(9.52)$ & 36 \\
\hline $\begin{array}{l}\text { Time from the prescription of actual } \\
\text { wheelchair(years) }\end{array}$ & $3.65(3.11)$ & 13.5 \\
\hline Cost of wheelchair & $2837.14 €$ & \\
\hline Qualitative Variables* & $\mathbf{N}$ & $\%$ \\
\hline $\begin{array}{l}\text { Professional that prescribes the Wheelchair: } \\
\text { Rehabilitation physician }\end{array}$ & 45 & $75 \%$ \\
\hline Previous assessment & 36 & $60 \%$ \\
\hline Training to use of wheelchair & 8 & $13.30 \%$ \\
\hline $\begin{array}{l}\text { Professional who does the training: Worker of } \\
\text { company }\end{array}$ & 5 & $8.30 \%$ \\
\hline Place to training: Hospital & 6 & $10 \%$ \\
\hline Acquisition of Wheelchair: Company & 44 & $73.30 \%$ \\
\hline Funding: Public Health Service & 53 & $83.30 \%$ \\
\hline
\end{tabular}

* Only the more frequent answers 


\section{Environmental factors}

Certain architectonical barriers also existed at their home $(\mathrm{N}=41)$, and previously 48 persons had made adjustments in different places of their residence. The analysis regarding the use of other assistive device showed a low frequency, as the $60 \%$ used only one or none device. AT for transfers only used by the $31.7 \%$ of sample, being the hoist $(n=16)$, and transfer table $(n=3)$, the most used. With respect to social context, it highlights that $83 \%$ are living with relatives, and only the $5 \%$ of sample didn't need the support from caregiver. For the rest, the $72 \%$ had a relative as a caregiver, while the $23 \%$ had a personal assistant. The most frequent type of activities that they needed support was household $(\mathrm{N}=56)$, follow that ADL ( $\mathrm{N}=49)$, and mobility in the community ( $\mathrm{N}=49)$.

\section{Impact of wheelchair in life of participants}

To finish and in order to determine how is the impact of wheelchair in life of people with NMD, PIADS tool was applied. The results show that wheelchair has a positive, but moderate, impact of quality of life of people with NMD. The mean score for each dimension of PIADS were: competence $(M=1.06, S D=0.83)$; Adaptability $(M=1.23, S D=1.04)$; Self-esteem $(M=0.92 ; S D=0.87)$ Nevertheless, if the results are analyzed according different variables, like type of wheelchair, frequency of use or independence in locomotion, there is a clear difference between groups and between mean of each group with respect to total sample. Figure 5 $\& 6$ show the differences in PIADS score for participants.

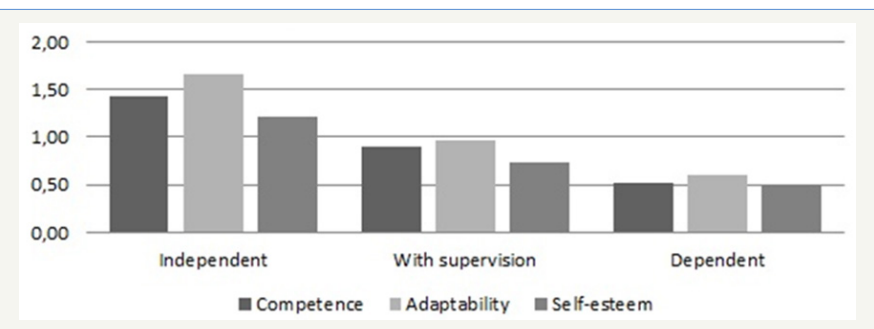

Figure 6: Score of PIADS depending of independence in locomotion.

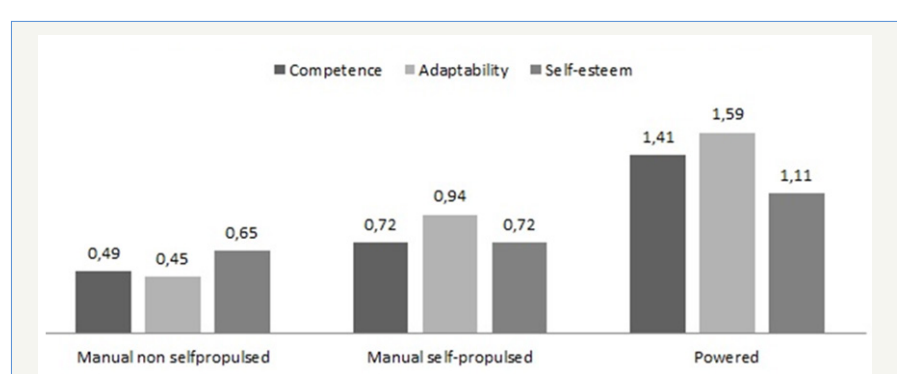

Figure 5: Score of PIADS depending of type of wheelchair.

It is necessary highlight that the main difference in PIADS' scores is due to type of wheelchair used. Persons who used a manual wheelchair had lower scores in the three dimensions (mean Competence $=0.67 ;$ adaptability $=0.84$; self-esteem $=0.7$ ), than those that used powered wheelchair (mean Competence $=1.41$; adaptability=1.59; self-esteem=1.11), $\mathrm{p}<0.01$. In order to know the possible influence of studied variables on that psychosocial impact of wheelchair, bivariate analyses were applied. In this case, Table 3 shows the difference means (for qualitative variables) and correlation coefficient (for quantitative variables) in dimensions of PIADS for those variables with significant influence.

\section{Discussion}

This study highlights the profile of socio-demographical characteristics, activity and participation, features of wheelchair and environmental factors of people with NMD, users of wheelchair. Moreover, research was a comprehensive investigation of the impact of wheelchair use on the quality of life of this population. Overall, the results showed that wheelchair use has a positive psychosocial impact. The progressive and chronic nature of NMDs produces a loss of functional capacity to perform certain activities, including those that require personal mobility. It is clearly necessary to prescribe wheelchairs for individuals whose ability to walk has been reduced or lost due to the disease [7,21-23]. Prescription of a wheelchair is an indication that the disease has progressed. During the initial adaptation phase users frequently report feeling a loss of control over their own capacities, performance and environment [24]. Choosing the appropriate moment to prescribe a wheelchair is a delicate process; if someone receives a wheelchair too early in the course of the disease and gets used to relying on it for personal mobility, there will be a more rapid loss of physical ability to walk, Muscular Dystrophy Campain [22].

The PIADS scores demonstrated that wheelchair use was associated with a positive impact on three aspects of psychosocial functioning: competence, adaptability and self-esteem. The greatest positive impact of wheelchair use was on adaptability, as in studies by Devitt et al. [25] \& Demers et al. [26]. This finding suggests that the empowerment to participate actively associated with wheelchair use is the most important factor for satisfaction of users with the wheelchair the smallest impact of wheelchair use was on self-esteem, which was also consistent with earlier studies. Devitt et al. [25] suggested that self-esteem may be negatively affected by the progressive, unpredictable nature of NMDs. Additionally; wheelchairs represent a visible sign of disability and are associated with social stigma that may have a negative influence on users' selfesteem. Even when a wheelchair is absolutely necessary to mobility wheelchair use does not necessarily have an impact (positive or negative) on the quality of life of person with NMD. Some factors, such as low usage frequency and limited walking ability, had very little influence on how wheelchair use affected the user's wellbeing (PIADS scores close to 0 ). These findings suggest that a period of adaptation to the device, during which it is integrated into the user's lifestyle, might increase its positive impact on quality of life. The influence of certain variables on the impact of wheelchair use was also explored in this research. These variables included personal factors, activities, participation in social activities, environmental factors and wheelchair characteristics.

All these factors are potential moderators of the strength and direction of the association between wheelchair use and its psychosocial impact. Level of ambulation has been shown to 
have a marked influence on adaptability. It follows from this that wheelchairs facilitate participation in social activities, because give users without functional ambulation independent mobility, which opens up a wider range of activities and occupations. Locomotors independence, assessed with the FIM 'locomotion' item was reported to be one of the main determinants of positive impact of a device on quality of life in a study of people with multiple sclerosis [26]. The sense of freedom that wheelchair use gives contributes to users' wellbeing, self-esteem and self-confidence and increases their interest in new activities and ability to take advantage of opportunities as well as their sense of competence and efficiency.

Using a wheelchair to carry out activities of daily living was associated with much greater independence in such activities, but this was not reflected in a difference in mean PIADS scores. This finding is consistent with other studies, including that of Nätterlund \& Alhström [22] who argued that personal autonomy in activities of daily living was not be a good predictor of quality of life. Environmental factors play an important role in the use of AT for personal mobility because getting around will be harder if the environment is not accessible or supportive, regardless of the quality and suitability of mobility at $[27,28]$. Nonetheless, in this study we found no significant differences in competence and adaptability scores associated with the presence of environmental adaptations or architectural barriers. The data did not allow us to evaluate effects of physical aspects of users' home environments. Social environment has a strong influence on how people with disability interpret their experiences [29]. In general, the participants in this research had good and supportive relationships with relatives and their main caregiver. Such support is very important, not only to carrying out activities of daily living, but because it contributes to the person's sense of control, wellbeing and quality of life [30,31].

It appears that having support from caregivers is more important to deriving benefit from a wheelchair than lack of environmental barriers. Analysis of associations between wheelchair characteristics and PIADS scores indicated that impact on self-esteem was not related to device variables. Competence was the PIADS dimension for which there was the highest number of significant interactions, indicating that the characteristics and usage profile of the wheelchair influence its impact on the productivity, efficiency and fulfillment of users with NMD. Despite the obvious advantages of the powered wheelchair and the various studies assessing its effectiveness [8,32-34], to date there has been no empirical demonstration that it offers more benefits to users than a manual wheelchair. Furthermore there has been no research specifically on wheelchair use in people with NMD. In this study use of a powered wheelchair was one of the main factors positively associated with greater impact of wheelchair use. This finding is consistent with the results of other studies of people with neurodegenerative diseases such as multiple sclerosis [25] and amyotrophic lateral sclerosis [21]. This study confirmed the conclusions of earlier research [35-38] which indicated that using a powered wheelchair improved independence with respect to mobility and social interaction and also improved the comfort and quality of life of people with NMD.

\section{The present study has few limitations, like the ones that we discuss below}

i. Low number of participants. That is due to the absence of official database or epidemiologic studies in Spain. Nevertheless, all persons (except 5 registers) from NGO's database that met inclusion criteria participated in the research. This limitation made it difficult to apply other analysis, like structural equation model.

ii. Uncontrolled variables: Researchers consider that certain individual characteristics could have not controlled, like possible presence of alterations in mood, the financial factors and social resources, or the level of assistance received from social-health services.

iii. Difficult to apply PIADS tool: During interviews, researchers perceived that participants had difficult to understand the mean of certain items. So, the questionnaire was applied with all definitions given.

Despite these limitations, the present study highlights valuable information about activity and participation's profile of people with NMD, characteristics of their used wheelchair and the impact in their life, in order to make recommendation to optimize the process of wheelchair's prescriptions for this population: assessment, training and follow-up process about use of wheelchair. It could be the starting point to improve the provision system of wheelchair and to include the best devices for people with neuromuscular disorders, according to the profile offered in this research, updating the national catalog of orthprothetics services.

Future research about this topic could be include to performance a longitudinal study to determine the effect of wheelchair or another AT's use, analyzing the time estimated to adapt to this AT, check the predisposition of this use and, finally, to follow-up the person's characteristic and new needs. On the other hand, to validate the real effects and influences from environmental factors, especially these related with physical, culture and social's contexts, could be interesting to reproduce this study in other regions of Spain and, in other countries, both European nations, and developing countries. This research can highlight and estimate the effect of that environmental factors to use (or not) the wheelchair in the social life. Finally, also could be interesting to carry out a comparative study between diagnosis groups, that allows analyze the influence of specific characteristics of each pathology and the differences with respect the possible influence psycho emotional skills.

\section{Conclusion}

The wheelchair becomes an additional part of the person affected with NMD. Consideration is given to a fundamental, environmental factor to maintain independence in activities as basic as mobility. Knowledge of how the wheelchair is incorporated into a user's daily life will help him/her to reach his/her objectives and expectations, and to establish the social and personal benefits derived from its use. Architectural barriers are a limiting environmental factor in the use of wheelchairs. Modification of the 
user's home is necessary in order for users with NMD to facilitate the usage of this assistive device. During the process of prescribing a wheelchair, the professional health care provider should consider not only the user but also his/her needs in terms of goals, fulfillment of activities, and degree of participation, as well as his/ her interaction with the environment. The use of PIADS measures has made it possible to consider the user's perspective, as well as his/her expectations, values and psychosocial characteristics.

The application of PIADS have facilitated the determination of both the effects of wheelchair usage by users with NMD as well as the factors that shed light on the impacts of such usage. Independence in personal mobility is the main factor determining a positive impact on the quality of life associated with wheelchair use. There is a period of adaptation in the use of the first wheelchair before a positive psychosocial impact is realized. During this phase, the user constructs an attributed significance to the device. The low level of usage of wheelchairs and the ability to maintain a residual degree of locomotion hardly affect how a user perceives the impact of this assistive device on quality of life. A wheelchair has a positive social impact in terms of the perceived quality of life in persons with NMD. Among the different types of assistive tools, the electronic wheelchair offers improved competency and adaptability in users. Practical knowledge of the actual requirements and the degree of satisfaction associated with the usage of wheelchairs by uses with NMD is fundamental to both meet the needs of this population of users as well as to boost the industries associated with these devices. Consequently, it is important to conduct studies, such as the one described here, which are aimed at clarifying the concrete, specific demands and needs of users in each user population.

\section{References}

1. Cup EH, Pieterse AJ, Knuijt S, Hendricks HT, van Engelen BG, et al. (2007) Referral of patients with neuromuscular disease to occupational therapy, physical therapy and speech therapy: usual practice versus multidisciplinary advice. Disabil Rehabil 29(9): 717-726.

2. Abresch RT, Han J, Carter GT (2009) Rehabilitation management of neuromuscular disease: the role of exercise training. J Clin Neuromuscul Dis 11(1): 7-21

3. McDonald CM (2002) Physical activity, health impairments and disability in neuromuscular disease. Am J Phys Med Rehabil 81(11): 108-120.

4. http://www.orpha.net/consor/cgibin/Disease_Search.php

5. Pieterse AJ, Cup EH, Knuijt S, Hendricks HT, van Engelen BG, et al. (2008) Development of a tool to guide referral of patients with neuromuscular disorders to allied health services. Part two. Disabil Rehabil 30(11): 855862.

6. Pousada García T, Groba González B, Nieto Rivero L, Pereira Loureiro J, Díez Villoria E, et al. (2015) Exploring the Psychosocial Impact of Wheelchair and Contextual Factors on Quality of Life of People with Neuromuscular Disorders. Assist Techno 27(4): 246-256.

7. Bostrom K, Natterlund BS, Ahlstrom G (2005) Sickness impact in people with muscular dystrophy: a longitudinal study over 10 years. Clin Rehabil 19(6): 686-694.

8. Richardson M, Frank AO (2009) Electric powered wheelchairs for those with muscular dystrophy: problems of posture, pain and deformity. Disabil Rehabil Assist Technol 4(3): 181-188.

9. Hamed R, Tariah H A, Hawamdeh ZM (2012) Personal factors affecting the daily functioning and well-being of patients with multiple sclerosis using the International Classification of Functioning model. Int J Ment Health 41(4): 47-61.

10. Louise Bender PT, Kim J, Weiner B (2002) The shaping of individual meanings assigned to assistive technology: a review of personal factors. Disabil Rehabil 24(3): 5-20.

11. Smith R (1991) Technological approaches to performance enhancement. In: Christiansen C, Baum C (Eds.), Occupational Therapy: overcoming human performance deficits. New Jersey, USA.

12. (1997) Uniform Data System for Medical. Functional Independence Measure, version 5.1 Buffalo General Hospital, New York, USA.

13. Linacre JM, Heinemann AW, Wright BD, Granger C V, Hamilton BB, et al. (1994) The structure and stability of the Functional Independence Measure. Arch Phys Med Rehabil 75(2): 127-132.

14. Sharrack B, Hughes RA, Soudain S, Dunn G (1999) The psychometric properties of clinical rating scales used in multiple sclerosis. Brain 122 (1): 141-159.

15. Dodds TA, Martin DP, Stolov WC, Deyo RA (1993) A validation of the functional independence measurement and its performance among rehabilitation inpatients. Arch Phys Med Rehabil 74(5): 531-536.

16. Rodíguez L (1997) Measure of Functional Independence (MIF). Guide for the use of the uniform data system for physical medicine and rehabilitation. Scientific Documentation Service of the FAES Group, Madrid, Spain.

17. Jutai J, Day H (2002) Psychosocial Impact of Assistive Devices Scale (PIADSC). Technol Disabil 14(2002): 107-111.

18. Day H, JW J (1996) Measuring the psychosocial impact of assistive devices: the PIADS. Can J Rehabil 9(2):159-168.

19. Jutai J, Gryfe P (1998) Psychological factors in the assessment of assistive technologies. In: Proceedings of RESNA '98. Minneapolis, USA: pp. 54-65.

20. Jutai J, Rigby P, Ryan S, Stickel S (2000) Psychosocial impact of electronic aids to daily living. Assist Technol 12(2):123-131.

21. Campaign MD (2011) Wheelchair provision for children and adults with muscular dystrophy and other neuromuscular conditions.

22. Natterlund B, Ahlstrom G (2001) Activities of daily living and quality of life in persons with muscular dystrophy. J Rehabil Med 33(5): 206-211.

23. Vandervelde L, Van den Bergh PY, Goemans N, Thonnard JL (2009) Activity limitations in patients with neuromuscular disorders: a responsiveness study of the ACTIVLIM questionnaire. Neuromuscul Disord 19(2): 99-103.

24. Asem F (2008) Guide to Neuromuscular Diseases. Information and ahoy to families. Formation Alcalá, Vol. 1, Jaen, Spain.

25. Devitt R, Chua B, JW J (2003) The Effect of Wheelchair Use on the Quality of Life of Persons with Multiple Sclerosis. Occup Ther Health Care 17(4): 63-79.

26. Demers L, Monett M, Descent M, Jutai J, Wolfsan C, et al. (2002) The Psychosocial Impact of Assistive Devices Scale (PIADS): translation and preliminary psychometric evaluation of a Canadian-French version. Qual Life Res 11(6): 583-592.

27. Harris F (2007) Conceptual issues in the measurement of participation among wheeled mobility device users. Disabil Rehabil Assist Technol 2(3): 137-148.

28. Salminen AL, Brandt A, Samuelsson K, Toytari O, Malmivaara A, et al. (2009) Mobility devices to promote activity and participation: a systematic review. J Rehabil Med 41(9): 697-706.

29. MJ S (2001) Assistive technology matching device and consumer for successful rehabilitation. American psychological Association, Washington DC, USA.

30. Boyer F, Novella JL, Coulon JM, Delmer F, Morrone I, et al. (2006) Family caregivers and hereditary muscular disorders: association between 
burden, quality of life and mental health. Ann Readapt Med Phys 49(1): 16-22.

31. Boyer F, Drame M, Morrone I, JL N (2006) Factors relating to career burden for families of persons with muscular dystrophy. J Rehabil Med 38(5): 309-315.

32. Im SH, Lee SC, Moon JH, Park ES, Park YG, et al. (2010) Quality of life for primary caregivers of muscular dystrophy patients in South Korea. Chin Med J (Engl) 123(4): 452-457.

33. Vega E (2007) Acquired brain damage. The invisible disability, pp. 7-9.

34. Davies A, De Souza LH, Frank AO (2003) Changes in the quality of life in severely disabled people following provision of powered indoor/ outdoor chairs. Disabil Rehabil 25(6): 286-290.

35. Evans S, Neophyte C, de Souza L, Frank AO (2007) Young people's experiences using electric powered indoor - outdoor wheelchairs
(EPIOCs): potential for enhancing users' development? Disabil Rehabil 29(16): 1281-1294.

36. Liu M, Mineo K, Hanayama K, Fujiwara T, Chino N, et al. (2003) Practical problems and management of seating through the clinical stages of Duchenne's muscular dystrophy. Arch Phys Med Rehabil 84(6): 818-124.

37. Frank AO, Ward J, Orwell NJ, McCullagh C, Belcher M, et al. (2000) Introduction of a new NHS electric-powered indoor/outdoor chair (EPIOC) service: benefits, risks and implications for prescribers. Clin Rehabil 14(6): 665-673.

38. (2007) Resolution of May 29, 2007, of the Secretary of State for University and Investigation, which grants of shares under the Program of Studies and Analysis, aimed at improving the quality of higher education 141: $25750-25753$. 